\title{
OLD VERSUS NEW: TECHNOLOGY AND NEW FORMS OF ARCHITECTURE
}

\author{
Shiva Pouryousef Khameneh ${ }^{1,2}$ \\ ${ }^{1}$ Oxford Brookes School of Architecture, Department of Interior Architecture, Oxford Brookes \\ University, Oxford, United Kingdom, England \\ ${ }^{2}$ Interior Design Department, Pratt Institute, New York, United States of America
}

\begin{abstract}
This research has been undertaken partly due to the literature review on this subject. Whilst it's accurate to say that there is much contentious debate on the subject of digital modelling and its place in architecture in general, the debate has lessened somewhat in the last decade or so, and the volume of more technically-minded books on the subject suggest a subtle shift away from the original debate of the role of digital modelling on interior architecture, and more towards a tacit acceptance of its presence as a tool for creative invention. This paper seeks to qualify the influence that digital modelling has had on interior architecture, particularly in recent years since its widespread adoption. In particular, it seeks to investigate new forms of interior architecture and design trends that would otherwise not have been possible without digital modelling programs. In order to accomplish this, hypotheses will be formed out of the author's personal experience and works, and a discussion and analysis relevant findings and arguments drawn from conclusions of other authors on the subject will be used to contextualise and frame the hypotheses to see if they stand up to scrutiny.
\end{abstract}

Keywords: digital modelling, architecture,form, technology

\section{INTRODUCTION}

"Is the proliferation of sophisticated tools for modeling, parametric design, and digital fabrication making the practice of sketching

by hand obsolete?"-Joann Gonchar (2012)

With the exponential increase in processing power over a very short period of time, the possibilities of digital modelling have increased in much the same way. For instance, according to Mara (2010), new possibilities in digital modelling, such as building information modelling (BIM) and solid modelling, are enticing architectural firms around the world. Tasli and Sagun (2002) have previously written about such exciting possibilities; with

regards to exteriors, an architect may be able to simulate the full life cycle of a building, building-user interactions, weather effects, and light sources. Mara (2010) writes that such information has been historically more relevant to 'engineers and manufacturers', but now architects can see the realtime effects of every line, every plane of their work. The ability to render light sources and shadows, and provide virtual walkthroughs of spaces, is of particular interest to interior designers. (Höhl, 2009)

However, even with this increased functionality, many architects and firms maintain that hand-drawn sketching remains a vital part of the creative process. According to Gonchar (2012), a Yale School of Architecture symposium in February 2012 asked the question 'Is Drawing Dead?' The responses were surprising. She writes:

"Finnish architect Juhani Pallasmaa, for instance, made the case for sketching as a tactile tool for discovery. While drawing, an architect isn't focused on the individual lines he or she is creating, but is instead "occupying that space, as if touching all its surfaces," said Pallasmaa. Such a kinesthetic connection is "difficult, if not impossible, to simulate with computers," he said."

In fact, the lecturer who organised the symposium, Victor Agran, did so not to pay some form of nostalgic homage to the art of hand-drawing, but to combat 'a loss of visual literacy in academia'. The implication here is that digital modelling has hampered the creative process. 
Peng (2001) makes some bold declarations in the beginning of his book, Design through Digital Interaction: Computing Communications and Collaboration on Design. He writes (2001, p.1):

"Seen from the dawn of the twenty-first century, the use of computers in architectural design can be traced back at least three decades ago. What we see from the past is that many earlier predictions or expectations of how computers might fundamentally change the ways we design buildings, or more broadly, the built environments, did not come to fruition for various reasons."

Sean Pickersgill of the University of South Australia is one of the only writers, certainly in recent years, to actually pick up on and note the impact that digital modelling has had on architecture as a whole. He writes (2010, p.336):

"There is little argument that the development of complex geometrical computing applications such as Autodesks' Max and Maya, and latterly Bentley Systems' Generative Components has brought about a sea change in the design and representation of architectural projects in recent years. These works, either in their digital or built format, constitute an emerging body of experience that exploits our familiarity with the virtual spatiality of these processes to create immersive experiences that to greater or lesser extent explore the narratival and ludological [sic] opportunities of these environments."

According to Andrew Barrie (2010), the 20th century saw a move away from traditional methods of thinking of the relationship between a building's exterior and its interior by separating the functions of the two - he argues that this is primarily due to the role played by Le Corbusier's modernist movement in calling for novel changes to architectural function. Key among these is the relationship between a building's exterior and its interior load supporting walls - before this movement, these were often integrated as the same thing, with the 'exterior envelope' functioning as a loadbearing system. In this approach, the interior design and function remained hidden and separate from the design and function of the exterior envelope. However, Le Corbusier's modernism promoted a separation of these functions, moving load-bearing walls and columns further towards the inside of the building, and letting some of the peripheral interior space interact with the building's exterior.

However, he notes that since the turn of the century, this concept has dramatically fallen out of favour, giving particular examples in Japan of Toyo Ito and Kazuyo Sejima, saying, "Ito and Sejima are like the moderns in that they have made use of the new materials and methods of their time, but they have effectively reversed the Modernist innovations, re-integrating structure and envelope with the aim of creating new types of space." (2010, p.40) He pins the genesis of this shift squarely on digital modelling; specifically, the structural analysis and innovation of materials afforded by new technology. He writes:

"New digital techniques for modelling and structural analysis allow the creation of structures whose performance was previously impossible to verify. Innovations in material technology and fabrication techniques (particularly digitally-controlled cutting and moulding) are making possible complex forms and structures that were previously prohibitively expensive or too technically demanding." (2010, p.40)

However, central to his premise is that technology has not developed for its own sake - "the impetus behind these developments is not technological" - but is rather a response to the demands of architects to be able to realise new concepts of space. He also talks about the aforementioned belief that these technologies have reduced costs associated with labour and materials. Furthermore, he suggests that this breaking of the mould represents a desire to break from modernism's grid-like rigidity and insert more complexity and individualism into their works, both interior and exterior. In this sense, he seems to be suggesting a feedback loop of sorts: digital technologies have informed new types of architectural ideas, which in turn have been the primary drive for the development of advances in that same digital technology, which in turn have allowed for even greater freedom to introduce complexity into new ideas, ad infinitum. 
What we see from Peng, Barrie and Pickersgill seems at first like a contradiction: people in the past believed that $\mathrm{CAD}$ would fundamentally alter the way in which architects design, whereas Pickersgill writes just nine years later that there is 'no question' that these technologies have brought about 'sea change in the design and representation of architectural projects'. It seems, therefore, that at least one of these authors is either mistaken, or has misspoken. The real question here is whether or not these technologies have changed the way we design, or changed what we design. This dichotomy is not explicitly or implicitly addressed by any of the authors. However, the literature has already revealed stark contrasts of opinions: the way we design has no doubt changed, at least in part. Even if traditional hand-drawn representations of concepts and ideas of space persist, there

would seem to be few architects today within any discipline or specialisation who would persist with hand-drawn and physical models throughout the entire design process. The merits of a mixed approach (for example, initial sketching and brainstorming carried out by hand, to be transferred to a digital medium to perfect and analyse the design) have been debated to some extent, and would seem to be a case of the architect's personal preference, either due to a lack of computing skills, a force of habit, or having been in practice for much longer than most computers have, as well as whether or not the space being designed is exterior or interior in nature. It is the case, however, that nowadays the final drawing of a project is presented from a computer. To underscore this point, David Derine's book Architectural Drawing (2010) gives architects advice on how to draw in

a variety of different media - however, it openly states:

"CGIs vary in character and complexity but this technique is now used for the vast majority of contemporary architectural renderings. More often than not the final image is made by working in a number of different software packages. Invariably these programs support a formal imagination and are at their best when describing complex forms, structural detail and photorealistic lighting that would otherwise be difficult to represent $[\ldots]$ the photorealism of CGI is something relatively new and, using a handful of software packages, this super-realistic render has become a global standard." (2010, p.33)

As a result, it is often much easier simply to design the project in a digital medium to facilitate the creation of the final architectural render. Derine writes:

"It can also be useful to develop sketch models digitally that are more quickly 'rendered'. In this sense SketchUp is a popular and useful tool. It is precise as well as being quick to use [...] Within SketchUp itself are useful guides to sciagraphy, material palettes and components; within Layout, orthogonal drawings can be quickly set up from the sketch model.” (2010, p.33)

Photoshop is also used to help add extra detail and tweak certain characteristics of architectural renders made from digital models.

It almost seems self-evident, even to those who would have no background in architecture, that designing by hand and on a computer are two different things, just as writing by hand and word processing each have their own advantages and disadvantages. Nevertheless, the debate on whether or not these technologies have impacted what we design is less certain. However, those that do tackle the topic tend to argue for the same key points:

- It is faster (a point of debate depending on the age of the source; early writings from 20 to 30 years ago tend to disagree, more modern ones emphatically agree, though only give anecdotal evidence to support the assertion)

\section{- It allows for more complexity in design}

- It allows for a greater range of analytical tools of a design (life-cycle and interaction modelling, structural soundness which in turn allows for alterations to the design, weather modelling, developing excellent CG renders of a space for promotional material, etc.)

- It is cheaper both in labour and materials, which leads to a better use of those materials 
Another subject not yet discussed is on the collective design process - most authors have written exclusively about architects as if they exist in their own bubble, being solely responsible for an entire project throughout its life. Peng (2001) charts the development of CAD, and what he calls the transition to Computer Supported Collaborative Design, whereby advances in information technology and networks have enabled a greater level of collaboration on projects. In addition, no authors address external influences on the adoption of digital modelling tools - for example, in many parts of the world, local planning authorities insist that all drawings and site plans submitted with a planning application are lodged online so that the authority can place the digital model of the proposed development within a large 3D map of the local area to check for things such as obstruction of light to other buildings and roads, and lines of sight.

Thus far, this paper has set out four hypotheses to be investigated based on personal work and experience; a comprehensive literature review has been carried out on the research question, and the results and arguments forming around certain concepts have been analysed and set out against those hypotheses in Chapter 4 . The aim of this chapter is to provide resolution to the analysis, set against each hypothesis, as well as to briefly describe limitations to this research.

\section{1: Digital modelling is no faster than drawing by hand}

The analysis of the literature review seems to neither support nor reject this hypothesis. In fact, the most important point taken from the authors is that it depends entirely on what the architect hopes to achieve with digital modelling software. In other words, many authors outright rejected the idea that digital modelling is useful in the initial design phase of a project, with the most common argument being that it impedes the creative process in conceptualising new and different ways of approaching a task or project. However, Penz's research showed that, when compared to hand drawing in the design and drafting of a project from start to finish, digital modelling was slightly slower (albeit on very dated technology). Because of this, I believe that this hypothesis is unique among the four in warranting further research, such as a replication of Penz's experiment with more modern technology, and in an environment today where most architects of all specialisations regularly use and have a solid working knowledge drafting with computers.

\section{2: Interior architects will continue to use a mix of media, and not exclusively use digital modelling throughout a project}

The resounding consensus shown in the analysis of this hypothesis is that virtually all of the writers make this point, typically by stating that digital modelling has the potential to impede the brainstorming nature of the concept design process. One recommendation for future research would be a large survey of architects in practice throughout the world measuring exactly how much of their work (as represented by time) is actually done by hand, and how much by computer.

\section{3: Digital modelling has created new styles and forms of interior and exterior space by being able to offer more tools to architects to realise more daring and technically challenging designs.} Only a few of the authors touched on this point, but those who did agreed. As discussed in the analysis, several questions beyond the analysis of the literature review were raised, such as the notion of the destruction of ideas, as well as the exact nature of the causation between digital modelling and new forms of space. However, the consensus formed around this topic indicates quite clearly that this hypothesis has been supported by the authors, and future research could be undertaken to establish the correlation between these two ideas, such as the survey recommended for 2 .

\section{4: Digital modelling offers cost benefits over traditional modelling methods}

This hypothesis was also fully supported by the literature analysis. It also raised the question of exactly how the authors back up these assertions; Porter mentions Chrysler's model development time reduction with the introduction of computer-controlled laser cutting. Future research could look into actual costs involved in the creation of projects, both past and present, to assemble concrete data. For 
example, Penz's test could be repeated, but with an added element monitoring for probable labour and materials costs.

\section{LIMITATIONS OF THE RESEARCH}

It was originally planned that this author would interview several practising architects to gauge their responses to a series of questions that were derived from the literature review. The intent was to add extra weight to the conclusions presented above. Due to the limits on the timescale of the research, and the fact that it was being written over December and January when most people are on annual leave, the architects who expressed an interest to take part were unable to complete the questionnaires in time. Though the underlying conclusions drawn from the literature review, most of which was written by architects in practice, would be unlikely to change, having the results of the interviews would have added additional primary data to the research.

\section{REFERENCES}

Barrie, A. (2010). “After Modern/After Imagination”, in Imagining: proceedings of the 27th international SAHANZ conference. 30 June - 2 July 2010.

Bertol, D., (1997). Designing Digital Space: An Architect's Guide to Virtual Reality. New York: John Wiley \& Sons

Black , A. (1990). The Monotype Publishing Guide and Glossary. London: The Design Council Dernie, D. (2010). Architectural Drawing. London: Laurence King Publishers Frazer, J., (1995). An Evolutionary Architecture: Themes VII. London: Architectural Association. Gonchar, J., (2012). "Is the proliferation of sophisticated tools for modeling, parametric design, and digital fabrication making the practice of sketching by hand obsolete?" in Architectural Record, v.200, n.4, pp.29-30.

Kolarevic, B. (2003). Architecture in the Digital Age: Design and Manufacturing. 1st ed. London: Spon Press Leach, N. (2009). Digital Cities AD: Architectural Design. New York: John Wiley \& Sons. Mara, F., (2010). “3D CAD Technology”, in Architects' journal, v.232, n.9, pp.33-36.

Peng, C. (2001). Design through Digital Interaction: Computing Communications and Collaboration on Design. Bristol: Intellect Books.

Penz, F., (1992). Computers in Architecture: Tools for Design. Hong Kong: Longman Group Ltd. Pickersgill, S. (2010). "Techniques of Digital Imagination: Vertigo", in Imagining: proceedings of the 27 th international SAHANZ conference. 30 June - 2 July (2010). Society of Architectural Historians, Australia and New Zealand. Porter, T. (2000). Architectural Supermodels. Oxford: Architectural Press. Silverman, D. (2009). Doing Qualitative Research. 3rd edition. London: SAGE Spankie, R. (2009). Basics Interior Architecture 3: Drawing Out the Interior. Lausanne: AVA Publishing SA. 